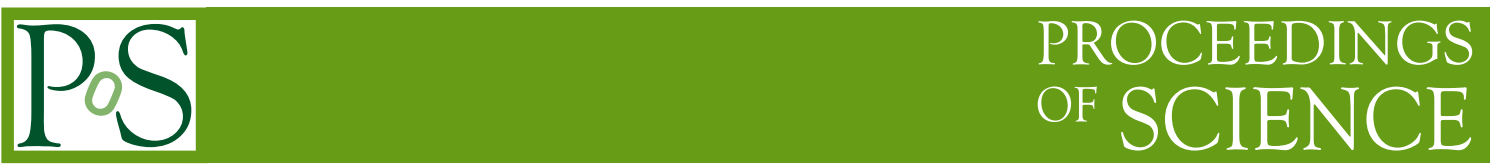

\title{
Directionality in Dark Matter search
}

\section{G. Fiorillo*}

Physics Department, Università degli Studi Federico II and INFN, Napoli 80126, Italy

Istituto Nazionale di Fisica Nucleare, Sezione di Napoli, Napoli 80126, Italy

E-mail: giuliana.fiorillo@na.infn.it

\section{Cadeddu}

Physics Department, Università degli Studi, Cagliari 09042, Italy

Istituto Nazionale di Fisica Nucleare, Sezione di Cagliari, Cagliari 09042, Italy

E-mail: matteo.cadeddudca.infn.it

\begin{abstract}
The potentialities of an argon Dark Matter detector with real-time directional information located at Laboratori Nazionali del Gran Sasso (LNGS) are discussed. Columnar recombination combined with a dual-phase Liquid Argon Time Projection Chamber (TPC) provides a promising approach for a directional detector. Even in the case of a minimal angular resolution, a very clear signature of WIMP scattering on target nuclei is shown to be expected. Indeed, the ratio of horizontal to vertical events changes by a factor of four during the day and has a characteristic sidereal-day periodicity. Demonstration of a directional sensitivity in a large Liquid Argon TPC could have high impact on the future experiments in the field, opening up the potential to find conclusive evidence for dark matter or disprove the WIMP hypothesis at and above the mass range explored by planned accelerator experiments.
\end{abstract}

Neutrino Oscillation Workshop

4 - 11 September, 2016

Otranto (Lecce, Italy)

*Speaker. 


\section{Introduction}

A plausible explanation of the nature of Dark Matter (DM) is that it is constituted by Weakly Interacting Massive Particles (WIMPs). The standard non-directional direct detection is very challenging in particular for what concerns background rejection. If detectors were able to distinguish the recoil direction of the scattered nucleus, the signal would have an unambiguous daily angular variation. As a consequence of the Earth's rotation around its axis, the WIMP apparent direction should in fact change during the day. Thus, the angular distribution of the recoil nuclei should vary with time. One of the most promising approach is a detector based on the so-called Columnar Recombination (CR) effect on a Noble Liquid [1]. In this detector, recoiling nuclei cause argon excitation and ionization. Each event is detected using argon de-excitation prompt scintillation light, $\mathrm{S} 1$, and the signal from free ionization electrons drifted by an electric field towards the top of the TPC, S2. A fraction of free electrons, however, recombine with ions. Models of CR suggest that the recombination probability should vary with the angle between the field and the track direction, modifying the amount of S1 and S2. A measurement of S1 and S2 may provide informations on the WIMP directionality. In order to produce a direction sensitive response the nuclear recoil must have an energy sufficient to form a track with definite direction [2]. Tantalizing hints of such a directional sensitivity in a LAr TPC have been reported in [3] above $57 \mathrm{keV}$. The results shown here are obtained considering an Earth-based detector, located at Laboratori Nazionali del Gran Sasso (LNGS), with a 20 tonne target mass of argon. This detector is assumed to discriminate only between tracks with different inclination relative to the vertical (electric field) direction. For this reason, the only measurable angle is the polar angle, $\theta_{r}$, while the azimuthal angle is integrated out.

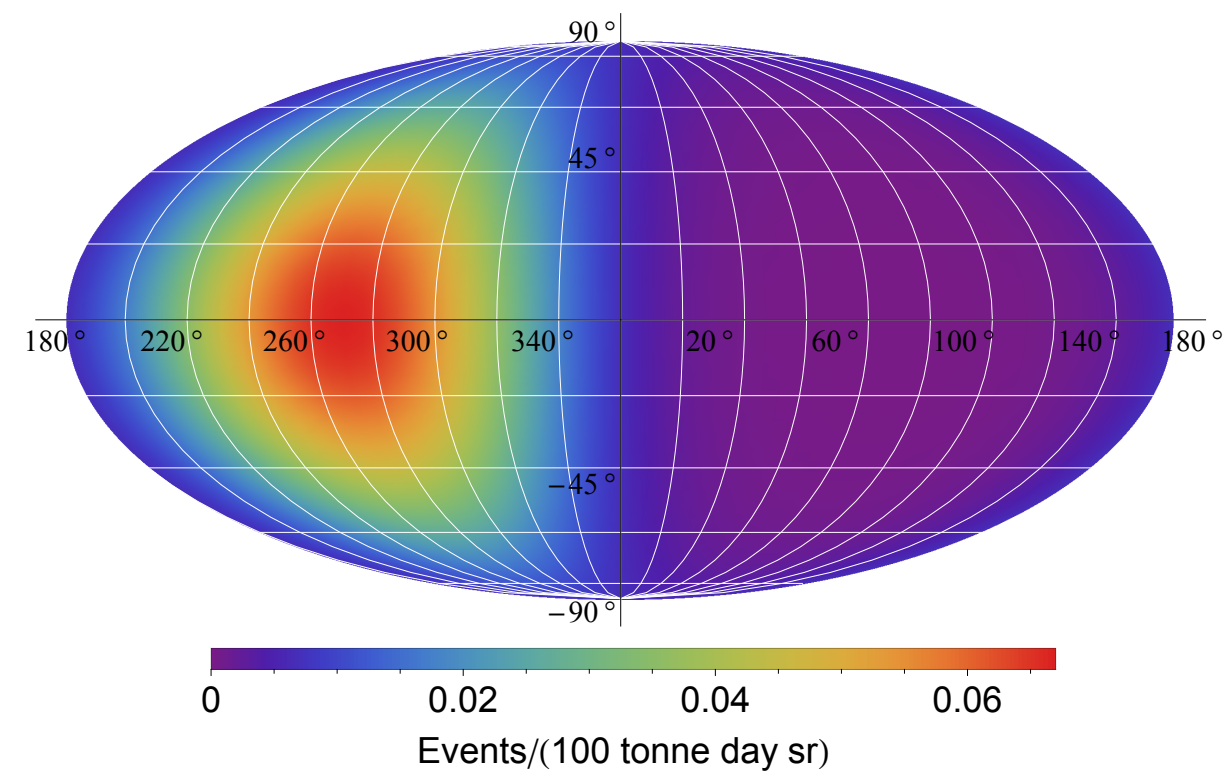

Figure 1: Directional differential recoil rate in argon integrated over the energy range $50 \div 200 \mathrm{keV}$, plotted in Mollweide equal area projection map of the celestial sphere in Galactic coordinates $(\ell, b)$. A WIMP mass of $200 \mathrm{GeV}$ and a WIMP-nucleon cross section of $10^{-46} \mathrm{~cm}^{2}$ is considered. 


\section{Recoil spectrum in galactic coordinates}

The general potentialities of a directional detector and, more specifically, the signature in the angular recoil rate of the detector motion through the WIMP wind are best illustrated in Galactic coordinates. In this coordinate system $\hat{x}$ points from the Sun towards the Galactic center, $\hat{y}$ in the direction of the Solar motion and $\hat{z}$ towards the Galactic north pole. In Fig. 1 it is shown the angular recoil rate

$$
\frac{d R\left(E_{t h}, E_{\max }, \hat{\mathbf{q}}\right)}{d \Omega_{r}}=\int_{E_{t h}}^{E_{\max }} d E_{r} \frac{d^{2} R\left(E_{r}, \hat{\mathbf{q}}\right)}{d E_{r} d \Omega_{r}},
$$

on a Mollweide equal area projection map in Galactic coordinates. The horizontal axis is the Galactic longitude, $0^{\circ}<\ell<360^{\circ}$, and the vertical axis the Galactic latitude, $-90^{\circ}<b<90^{\circ}$. The recoil rate is clearly anisotropic and points towards coordinates $\left(\ell=270^{\circ}, b=0^{\circ}\right)$, opposite to the direction of the Sun motion throughout the Galaxy.

\section{Results}

Here the clean advantage of a directional detection capability is highlighted. Events are divided in two classes. Horizontal events (HOR), defined by $\left|\cos \theta_{r}\right|<0.5$, and vertical events (VER=UP+DOWN), defined by $\left|\cos \theta_{r}\right|>0.5$, as shown in the left panel of Fig. 2. The ratio, R, of HOR and VER events is defined, which should be constant and equal to one for an isotropic signal. On the right panel of Fig. 2 the time dependence of this ratio and of the single components is shown. The thin lines represent the daily variation of the HOR and VER event rates, and their ratio, $\mathrm{R}$, for four equidistant sidereal days, while the corresponding blue (orange) bands represent the overall variation registered in the HOR (VER) component among different sidereal days of the year. The thick curves represent the averages over different sidereal days. Considering the annual
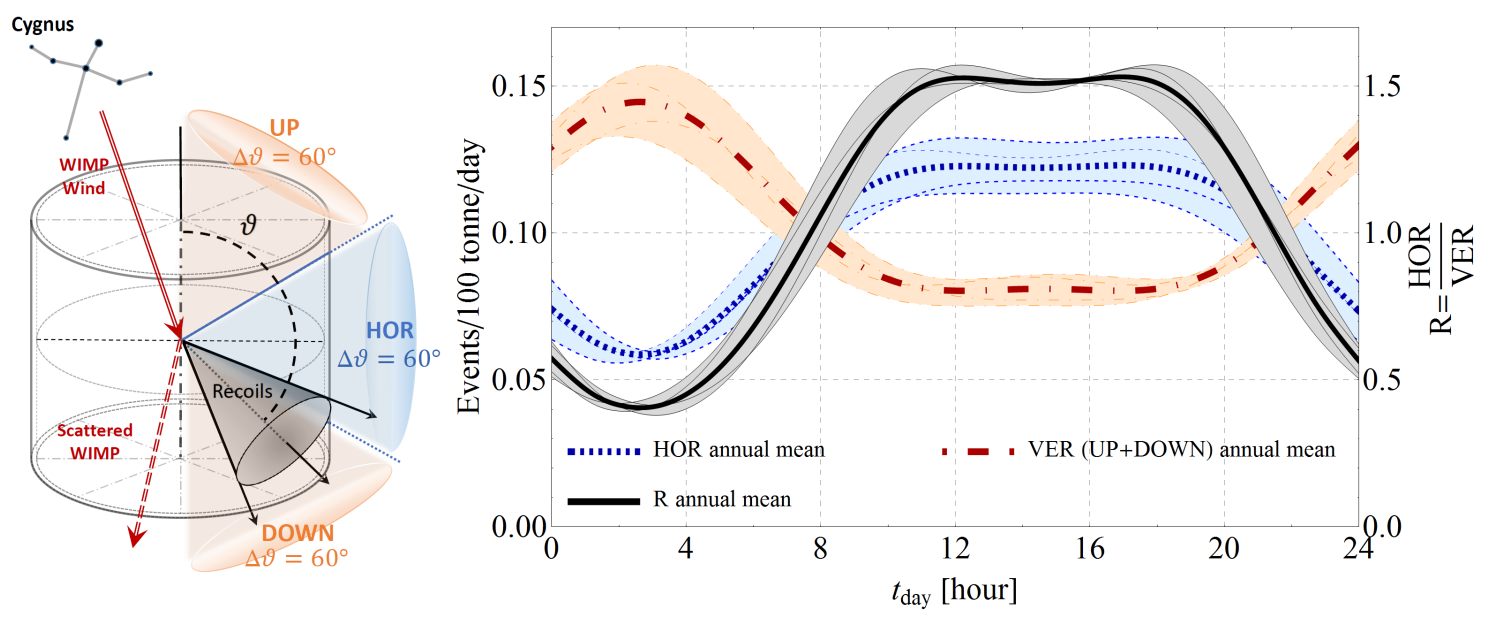

Figure 2: Left: Schematic representation of the recoils distribution between horizontal (HOR) and vertical (VER) events in a LAr TPC. Right: Daily variation of HOR (VER) event rate in blue dashed (orange dotdashed) lines for different sidereal days of the year. See text for details.

mean, the variation of the single component is of the order of $35 \%$ (29\%), with respect to the average daily value. For comparison, the seasonal modulation of the events that can be observed under 
the same assumptions, for the case of a non-directional WIMP detection is only about $8 \%$. Compared to it, the effect of the daily variation in a directional detector, even for this minimal angular resolution, confirms that adding the directional information increases significantly the discovery potential of a WIMP signature. Moreover, the annual mean ratio, $\mathrm{R}$, shows a remarkable deviation from one since it varies during the day by a factor $f \approx 4$.

To conclude, in these proceedings it is discussed how a directional detector located at LNGS could provide a very clear signature for WIMP signal. This signature is sizeable even for a minimal angular resolution. In particular, the daily modulation shown in Fig. 2 is an order of magnitude larger than the annual modulation obtained ignoring the angular information. Thus, a realistic detector able to use the angular information to reject the background would provide a strong evidence of DM, the latter due to the fact that no known background is correlated with the sidereal period.

\section{References}

[1] G. Jaffé, Ann. Phys. (Berlin) 347, 303 (1913).

[2] D. R. Nygren, J. Physics. Conf. Ser. 460, 012006 (2013).

[3] H. Cao et al., (The SCENE Collaboration), Phys. Rev., D91, 092007 (2015). 